(อ) OPEN ACCESS

\title{
Meropenem-induced thrombocytopenia: a paediatric case
}

\author{
Joanna Cachia (10, Paul Torpiano, David Pace
}

Department of Paediatrics, Mater Dei Hospital, Msida, Malta

\section{Correspondence to} Dr Joanna Cachia; joanna.cachia@gmail.com

Accepted 12 August 2021

Check for updates

(c) BMJ Publishing Group Limited 2021. Re-use permitted under CC BY-NC. No commercial re-use. See rights and permissions. Published by BMJ.

To cite: Cachia J,

Torpiano P, Pace D. BMJ

Case Rep 2021:14:e243443.

doi:10.1136/bcr-2021-

243443

\section{SUMMARY}

Meropenem is a broad-spectrum carbapenem widely used to treat both Gram-positive and negative bacterial infections, including extended-spectrum beta-lactamaseproducing microbes. We describe the occurrence of thrombocytopenia and hypersensitivity in a boy receiving intravenous meropenem for intra-abdominal sepsis secondary to perforated appendicitis. The patient developed a pruritic maculopapular rash with occasional petechiae, associated with severe thrombocytopenia, after 7 days of meropenem administration. Investigations for other causes of thrombocytopenia, including possible line sepsis, were unfruitful, and the thrombocytopenia did not resolve until cessation of meropenem. Druginduced reactions should be considered in children receiving meropenem who present with a rash and thrombocytopenia.

\section{BACKGROUND}

Meropenem is a widely prescribed broad-spectrum antimicrobial belonging to the carbapenem family. It has activity against Gram-positive and Gramnegative micro-organisms, as well as anaerobes and extended-spectrum beta-lactamase-producing microbes. Indications for use include septicaemia and complicated infections of the abdomen, skin, respiratory tract and urinary tract, making it a widely prescribed antibiotic in the hospital setting. Common side effects include nausea, vomiting and diarrhoea, while unusual side effects include skin hypersensitivity reactions. ${ }^{1}$ Here we report a rare occurrence of meropenem-induced thrombocytopenia and hypersensitivity in an 11-year-old boy. To our knowledge, such a reaction to meropenem has not yet been specifically documented in the paediatric setting. ${ }^{2}$ Although rare, drug-induced thrombocytopenia can quickly lead to life-threatening complications if not adequately addressed in a timely manner. ${ }^{3}$ We explore the differential diagnoses, investigations and management options used and described in the literature pertaining to this adverse event.

\section{CASE PRESENTATION}

A previously healthy 11-year-old boy was admitted with a 1-day history of severe abdominal pain, initially generalised and subsequently localising to the right iliac fossa (RIF). He was diagnosed with acute appendicitis and underwent emergency laparoscopic appendectomy, at which a perforated gangrenous appendix was resected successfully. Postoperatively, the child was treated with intravenous cefuroxime, gentamicin and metronidazole for possible intra-abdominal sepsis complicating the perforated appendicitis.

Despite exhibiting significant clinical improvement postoperatively, the patient's $\mathrm{C}$ reactive protein was noted to be persistently raised at $281 \mathrm{mg} / \mathrm{L}$ (five times the baseline) on day 5 postappendectomy, with recurrence of fever of up to $38^{\circ} \mathrm{C}$ recorded on day 8. Abdominal ultrasonography revealed a hypoechoic heterogenous fluid collection in the RIF, measuring around $4 \mathrm{~cm}$ by $2 \mathrm{~cm}$ on day 8 postappendectomy. The collection was deemed too small to be amenable to radiological or surgical aspiration. Cefuroxime and gentamicin were thus switched to intravenous cefotaxime, and metronidazole was continued. In view of persistent abdominal pain and fever, CT imaging of the abdomen was done on day 13 postappendectomy, confirming a persistent collection in the periappendicular space.

Ultrasound-guided drainage of the fluid collection was performed on day 14 postappendectomy. Extended-spectrum beta-lactamase-producing and carbapenem-resistant Klebsiella pneumoniae, as well as Enterococcus faecalis and Enterococcus avium, was cultivated from the drained fluid in the periappendicular space. The Klebsiella isolate was resistant to ertapenem but sensitive to meropenem. A peripherally inserted central catheter (PICC) was placed and treatment as changed to high-dose intravenous meropenem $40 \mathrm{mg} / \mathrm{kg}$ three times a day and teicoplanin on day 20 postappendectomy. The patient demonstrated sustained clinical improvement with defervescence thereafter, with serial ultrasonography showing gradual resolution of the intra-abdominal fluid collection.

On day 7 of intravenous meropenem administration (day 27 postappendectomy), a rapidly spreading pruritic, non-tender maculopapular blanching rash with occasional petechiae was noted to have developed over the child's trunk and upper limbs associated with recrudescence of fever up to $38^{\circ} \mathrm{C}$.

\section{INVESTIGATIONS}

Serial complete blood counts revealed progressively worsening isolated thrombocytopenia with a drop to $101 \times 10^{9} / \mathrm{L}$ from $322 \times 10^{9} / \mathrm{L}$ coinciding with the appearance of the rash and fever on day 7 of meropenem administration, and falling to $14 \times 10^{9} / \mathrm{L}$ on day 8 of intravenous meropenem. This was associated with a mild rise in C reactive protein $(30 \mathrm{mg} / \mathrm{L})$, though there was no derangement of the other cell lines, including leucocytes, and haematinics. Activated partial thromboplastin time ratio, international normalised ratio and fibrinogen assay were within normal limits. Repeat abdominal CT showed improvement of the 
RIF collection consistent with continued response to treatment. While the PICC line was removed in view of possible line sepsis, line tip and blood cultures were negative, as were stool and urine cultures. Repeated viral serology and molecular diagnostic testing were also taken and remained persistently negative.

\section{DIFFERENTIAL DIAGNOSIS}

Causes of acquired thrombocytopenia in children can be classified according to the underlying pathology. Thrombocytopenia can result from either decreased platelet production, increased peripheral platelet destruction, redistribution or laboratory artefact. Causes of decreased platelet production include viral infection (such as Epstein-Barr virus, cytomegalovirus and parvovirus); bacterial sepsis; nutritional deficiencies; bone marrow suppression secondary to chemotherapy and toxins; aplastic anaemia; and bone marrow infiltration as occurs in myelodysplastic syndrome and lymphoproliferative disease. Increased peripheral platelet destruction may result from disseminated intravascular coagulation (DIC), immune thrombocytopenic purpura (ITP), thrombotic thrombocytopenic purpura and autoimmune disorders such as systemic lupus erythematosus. Redistributive causes include haemodilution resulting from large volume resuscitation, and sequestration as in splenomegaly and portal hypertension secondary to liver cirrhosis. ${ }^{45}$

The process of narrowing down the aforementioned differential diagnoses required multiple serial investigations. Spurious thrombocytopenia, which occurs due to clumping of platelets during analysis, was confidently ruled out by low platelet count being repeatedly confirmed on serial blood counts. Viral causes were excluded on the basis of negative viral serology and molecular diagnostic testing for Epstein-Barr virus, cytomegalovirus and parvovirus. The benign clinical picture, stable vital signs, normal coagulation screen, minimally raised $\mathrm{C}$ reactive protein and normal white cell count, as well as negative blood, urine and faecal cultures, argued against sepsis and DIC as likely causes for acute thrombocytopenia in this case. The absence of clinically evident infection at the PICC line insertion site, together with negative PICC line tip and blood cultures, also discounted line sepsis as a possible cause.

Aplastic anaemia and lymphoproliferative diseases such as acute leukaemia were confidently excluded early in the differential due to normal blood films as well as the absence of derangement of erythropoietic, myeloid and lymphoid cell lines. In addition, lack of hepatosplenomegaly, lymphadenopathy and history of recurrent opportunistic infections further argued against aplastic anaemia or malignant processes. Absence of splenomegaly also ruled out sequestration and portal hypertension as causes. Furthermore, the child's normal growth parameters, haematinics and good socioeconomic background discounted nutritional deficiency as a possible cause of thrombocytopenia. ${ }^{45}$

Despite the presence of fever, autoimmune disorders, such as systemic lupus erythematosus, were also ruled out on the basis of the absence of other systemic signs or symptoms such as arthralgia, along with the presence of normal cell lines other than thrombocytopenia. Thrombotic thrombocytopenic purpura usually manifests as a pentad of fever, neurological signs, renal failure, anaemia and thrombocytopenia. As such, it was considered highly unlikely to be the cause in this case in view of normal neurological examination and renal function together with normal haemoglobin levels. ${ }^{4}$ Postoperative thrombocytopenia was ruled out as it tends to develop more acutely within 4 days of surgery and usually results from haemodilution. ${ }^{6}$
The two main differential diagnoses were thus drug-induced thrombocytopenia and ITP. Both are diagnoses of exclusion, and there is no reliable readily available single confirmatory laboratory test for either of these pathologies. ${ }^{7}$ In our case, thrombocytopenia was noted to have developed within 1 week of starting intravenous meropenem and teicoplanin, consistent with a possible diagnosis of drug-induced thrombocytopenia. The associated evidence of hypersensitivity in the form of a pruritic maculopapular rash and fever pointed away from ITP and towards a drug-related cause. The resolution postcessation of meropenem, without the concomitant need to administer steroids and intravenous immunoglobulins, further confirmed drug-induced thrombocytopenia secondary to meropenem treatment as the underlying cause. ${ }^{8}$ While thrombocytopenia with teicoplanin treatment has also been reported, this is exceptionally rare, and the improving platelet count despite ongoing teicoplanin argues against teicoplanin as the cause of thrombocytopenia in this case. ${ }^{9-11}$

\section{TREATMENT}

The timing of onset of thrombocytopenia after starting meropenem and evidence of hypersensitivity prompted discontinuation of intravenous meropenem on day 8 of treatment (day 28 postappendectomy). The child was treated with intravenous chlorphenamine for the intense pruritus. The rash resolved over the following 2 days, and following transfusion of a single unit of platelets after the platelet count fell to $2 \times 10^{9} / \mathrm{L}$ (day 30 postappendicectomy), the patient subsequently exhibited a gradual rise in the platelet count on serial blood count monitoring. Teicoplanin was continued, and based on the culture and sensitivity results from the intra-abdominal fluid collection, co-trimoxazole was initiated instead of the meropenem.

\section{OUTCOME AND FOLLOW-UP}

Fever resolved within 24 hours of cessation of meropenem, and the child remained afebrile and systemically well. He was discharged from hospital on day 35 postappendectomy with a platelet count of $471 \times 10^{9} / \mathrm{L}$. The boy is currently being followed up annually for monitoring of growth and platelet counts. No further intra-abdominal or haematological complications have been reported thus far.

\section{DISCUSSION}

Thrombocytopenia in children is defined as a platelet count of less than $150 \times 10^{9} / \mathrm{L}^{5}$ Drug-induced thrombocytopenia often manifests as an acute severe drop in platelet count, typically occuring within 1 week following initiation of drug administration, with platelet counts frequently falling below $50 \times 10^{9} / \mathrm{L}$, as observed in this case. ${ }^{12}$ Systemic symptoms such as fever, chills, nausea and vomiting often predate hypersensitivity skin reactions, bruising, ecchymoses or petechiae. These typically resolve around 1-2 days after the offending agent is withdrawn and diagnosis is often reached after excluding other possible relevant causes of thrombocyopenia. ${ }^{13} 14$

Non-immune and immune mechanisms have been suggested for drug-induced thrombocytopenia. Non-immune mechanisms include impaired maturation or replication of megakaryocytes and reduced release of platelets from the bone marrow. ${ }^{12} 1516$ Where an immune mechanism is involved, increased rate of apoptosis or increased peripheral destruction of platelets is implicated, and antibodies specific to individual drug structures have been identified. Such antibodies may naturally occur as immunoglobulins with predilection for epitopes on glycoproteins found 
on the platelet membranes. In the absence of the specific drug, these are not sufficiently reactive to cause platelet destruction, but when the implicated drug is introduced, it serves as a reversible binder of the antibody to the platelet glycoproteins, most commonly glycoprotein IIb-IIIa and glycoprotein Ib-V-IX. ${ }^{17}$

Drug-induced platelet antibodies targeting glycoproteins are most commonly detectable by enzyme immunoassay, flow cytometry or monoclonal antibody immobilisation of platelet antigens. Platelet antibody testing may hence be diagnostic of meropenemassociated drug-induced thrombocytopenia. ${ }^{18}$ However, glycoprotein assays have been found to have low sensitivity and high specificity. This implies that serological testing is useful to rule in but not rule out immune causes of thrombocytopenia, limiting its diagnostic utility. This may be due to the fact that these immune conditions are caused by various mechanisms, some of which are not solely secondary to platelet autoantibody formation. Antibody testing is thus arguably still of limited clinical utility. ${ }^{79}$ Furthermore, testing is not widely accessible and and standardised testing protocols are not uniformly adopted. ${ }^{20}$ For these reasons, platelet autoantibody testing was not employed in this case. In preference, a combination of clinical and laboratory features was used to elucidate the final clinical diagnosis of meropenem-induced thrombocytopenia.

Meropenem hypersensitivity is rare, with serious hypersensitivity and adverse haematological effects being reported in less than $1 \%$ of patients. ${ }^{21}$ These represent serious and potentially life-threatening complications. ${ }^{18}$ The association of meropenem with isolated thrombocytopenia seems particularly rare and is confined only to case reports or case series. ${ }^{3}$ In infants and children, no such case has been reported in the literature thus far. ${ }^{2}$ In adults, meropenem-induced thrombocytopenia has been reported in two case reports and in a study on meropenem side effects in a teaching hospital in Pakistan. ${ }^{18} 2122$ In one case report, a 59-year-old man developed severe thrombocytopenia 8 days after initiation of meropenem treatment. After ruling out other causes of thrombocytopenia and with positive platelet antibody testing, meropenem administration was stopped and restoration of platelet count was noted soon after. ${ }^{18}$ In the other case report, a 57-year-old man with a medical history of diabetes, hypertension and chronic kidney disease received standard meropenem dosing following an upper gastrointestinal bleed and developed thrombocytopenia 1 day after administration. No further bleeding episodes were observed and meropenem was stopped after 3 days, with restoration of platelet count within 5 days. Platelet antibody testing was not carried out in this case. ${ }^{21}$ In the study conducted by Khan et al, correlation of meropenem side effects with renal function was studied in a Pakistani teaching hospital. Thrombocytopenia was observed in $37.81 \%$ of patients receiving the drug, with a linear increase in side-effect profile observed with worsening renal function. ${ }^{22}$ In the second case report described, the patient's meropenem dose was not adjusted for the deranged renal function. This could explain the earlier onset of thrombocytopenia in this case.

Similarly rare is the association of meropenem with pancytopenia, with only three case reports identified after an extensive search. Pancytopenia was described in a preterm neonate, a 3-year-old child with a head injury and another child (age not specified) who had undergone liver transplantation. In all cases, derangement of all cell lineages was noted to develop in a staggered fashion, with thrombocytopenia predating drops in haemoglobin and absolute neutrophil counts. Derangements were noted over a range of 3 days -2 weeks after initiation of meropenem. Restoration of normal cell counts was obtained after stopping treatment, without immediate life-threatening complications. In none of these cases was platelet antibody testing performed. ${ }^{23-25}$

In most cases of drug-induced thrombocytopenia, cessation of treatment is sufficient for subsequent resolution of hypersensitivity and restoration of platelet count. Prognosis is generally deemed quite favourable. ${ }^{12} 1516$ Corticosteroids have occasionally been used in cases where ITP could not be confidently excluded in the differential diagnosis. In our case, corticosteroids were not administered in view of the rapidity by which thrombocytopenia improved after cessation of meropenem, as well as due to initial concerns about possible sepsis secondary to multidrugresistant organisms, which was in turn eventually ruled out. Drug-induced platelet antibodies have been shown to persist for years after exposure, indicating that the causative drug might cause further reactions if reinstated in future. ${ }^{14}$ Platelet transfusions in drug-induced thrombocytopenia are mostly indicated to control overt haemorrhage or when thrombocytopenia is severe enough to risk spontaneous haemorrhage (platelet counts of less than $10 \times 10^{9} / \mathrm{L}$ ). Transfusions, however, may be ineffective if the drug or its metabolites have not yet been cleared. ${ }^{26-28}$ The patient reported here had a platelet transfusion in view of severe thrombocytopenia and an already previously complicated postoperative course.

\section{Learning points}

- Review of patient medication for sources of adverse drug events should be an essential part of the diagnostic work-up of patients who present with new onset of rashes and cell line derangements.

- The association of meropenem with thrombocytopenia, although rare, should be considered in children with an acute severe drop in platelet count after exclusion of other pathologies.

- Management of drug-induced thrombocytopenia varies according to severity and risk of haemorrhage. Immediate recognition of the reaction and prompt cessation of the drug are necessary in order to prevent development of lifethreatening complications.

- Use of antimicrobials should always be objectively justified and follow approved guidelines to minimise morbidity and mortality from adverse drug events.

Contributors $J C$ contributed to investigation, writing of the original draft, as well as editing and visualisation of the manuscript after a review by other coauthors. PT contributed to conceptualisation, methodology, resource acquisition, investigation and data interpretation, as well as review and editing of the original draft. DP contributed to conceptualisation, investigation and data interpretation, review and editing of the original draft, as well as overall supervision of the manuscript preparation.

Funding The authors have not declared a specific grant for this research from any funding agency in the public, commercial or not-for-profit sectors.

Competing interests None declared.

Patient consent for publication Parents/guardian consent obtained.

Provenance and peer review Not commissioned; externally peer reviewed.

Open access This is an open access article distributed in accordance with the Creative Commons Attribution Non Commercial (CC BY-NC 4.0) license, which permits others to distribute, remix, adapt, build upon this work non-commercially, and license their derivative works on different terms, provided the original work is properly cited and the use is non-commercial. See: http://creativecommons.org/ licenses/by-nc/4.0/.

\section{ORCID iD}

Joanna Cachia http://orcid.org/0000-0003-3114-3277 


\section{REFERENCES}

1 Baldwin CM, Lyseng-Williamson KA, Keam SJ. Meropenem: a review of its use in the treatment of serious bacterial infections. Drugs 2008;68:803-38.

2 Maria Pacifici G. Clinical pharmacology of meropenem in infants and children. Clin Med Invest 2019;4:1-9.

3 Johansen ME, Jensen J-U, Bestle MH, et al. The potential of antimicrobials to induce thrombocytopenia in critically ill patients: data from a randomized controlled trial. PLoS One 2013;8:e81477.

4 Kaplan RN, Bussel JB. Differential diagnosis and management of thrombocytopenia in childhood. Pediatr Clin North Am 2004;51:1109-40.

5 Gauer RL, Braun MM. Thrombocytopenia. Am Fam Physician 2012;85:612-22.

6 Skeith L, Baumann Kreuziger L, Crowther MA, et al. A practical approach to evaluating postoperative thrombocytopenia. Blood Adv 2020;4:776-83.

7 Vrbensky JR, Moore JE, Arnold DM, et al. The sensitivity and specificity of platelet autoantibody testing in immune thrombocytopenia: a systematic review and metaanalysis of a diagnostic test. J Thromb Haemost 2019;17:787-94.

8 Onisâi M, Vlădăreanu A-M, Spînu A, et al. Idiopathic thrombocytopenic purpura (ITP) new era for an old disease. Rom J Intern Med 2019;57:273-83.

9 Agnelli G, Longetti M, Guerciolini R, et al. Effects of the new glycopeptide antibiotic teicoplanin on platelet function and blood coagulation. Antimicrob Agents Chemother 1987;31:1609-12.

10 Terol MJ, Sierra J, Gatell JM, et al. Thrombocytopenia due to use of teicoplanin. Clin Infect Dis 1993;17:927.

11 Veldman RG, van der Pijl JW, Claas FH. Teicoplanin-induced thrombocytopenia. Nephron 1996;73:721-2.

12 Danese E, Montagnana M, Favaloro EJ, et al. Drug-induced thrombocytopenia: mechanisms and laboratory diagnostics. Semin Thromb Hemost 2020;46:264-74.

13 Aster RH, Bougie DW. Drug-induced immune thrombocytopenia. N Engl J Med 2007;357:580-7.

14 Aster RH, Curtis BR, McFarland JG, et al. Drug-induced immune thrombocytopenia: pathogenesis, diagnosis, and management. J Thromb Haemost 2009;7:911-8.
15 Savage-Elliott I, Wu VJ, Sanchez FL. Drug-induced thrombocytopenia secondary to commonly used antibiotics in total joint arthroplasty. Arthroplast Today 2020;6:137-40.

16 Narayanan PK, Li N. In vitro monocyte/macrophage phagocytosis assay for the prediction of drug-induced thrombocytopenia. Curr Protoc Toxicol 2019;79:e68.

17 George JN, Aster RH. Drug-induced thrombocytopenia: pathogenesis, evaluation, and management. Hematology 2009;2009:153-8.

18 Huang R, Cai G-Q, Zhang J-H, et al. Meropenem-induced immune thrombocytopenia and the diagnostic process of laboratory testing. Transfusion 2017;57:2715-9.

19 Schmidt DE, Lakerveld AJ, Heitink-Pollé KMJ, et al. Anti-platelet antibody immunoassays in childhood immune thrombocytopenia: a systematic review. Vox Sang 2020;115:323-33.

20 Arnold DM, Kukaswadia S, Nazi I, et al. A systematic evaluation of laboratory testing for drug-induced immune thrombocytopenia. J Thromb Haemost 2013;11:169-76.

21 Dhande P, Deshmukh S. Acute thrombocytopenia due to meropenem: a case report. Int J Basic Clin Pharmacol 2019;8:2766.

22 Khan MU, Yousuf RI, Shoaib MH. Drug utilization evaluation of meropenem and correlation of side effects with renal status of patients in a teaching based Hospital. Pak J Pharm Sci 2014;27:1503-8.

23 Hussain K, Salat MS, Mohammad N, et al. Meropenem-induced pancytopenia in a preterm neonate: a case report. J Med Case Rep 2021;15:25.

24 Estella J, Villanueva J, Calvo M, et al. Bone marrow aplasia and meropenem in a paediatric patient. Br J Haematol 2000;111:984-5.

25 Pérez-Gorricho B, Zapardiel Ferrero J, Grupo de Estudio de Meropenem en Pediatría. [Meropenem as empirical therapy in moderate to severe infections in hospitalized children aged 3 to 12 months and 1 to 14 years]. Rev Esp Quimioter 2004;17:341-9.

26 Bakchoul T, Marini I. Drug-associated thrombocytopenia. Hematology Am Soc Hematol Educ Program 2018;2018:576-83.

27 Ho-Tin-Noé B, Jadoui S. Spontaneous bleeding in thrombocytopenia: is it really spontaneous? Transfus Clin Biol 2018;25:210-6.

28 Kenney B, Stack G. Drug-induced thrombocytopenia. Arch Pathol Lab Med 2009;133:309-14.

Copyright 2021 BMJ Publishing Group. All rights reserved. For permission to reuse any of this content visit https://www.bmj.com/company/products-services/rights-and-licensing/permissions/

BMJ Case Report Fellows may re-use this article for personal use and teaching without any further permission.

Become a Fellow of BMJ Case Reports today and you can:

- Submit as many cases as you like

- Enjoy fast sympathetic peer review and rapid publication of accepted articles

- Access all the published articles

- Re-use any of the published material for personal use and teaching without further permission

Customer Service

If you have any further queries about your subscription, please contact our customer services team on +44 (0) 2071111105 or via email at support@bmj.com.

Visit casereports.bmj.com for more articles like this and to become a Fellow 